\title{
On a fixed point theorem for mappings satisfying a contractive condition of rational type on a partially ordered S-metric space
}

\author{
Mohammed Sani Mashina \\ ${ }^{1}$ Department of Mathematics Ahmadu Bello University, Zaria, Nigeria. \\ mohsanmash@gmail.com
}

Keywords: Contractive mapping, fixed point, Partially ordered set, S-metric space.

Abstract. In this paper we prove a fixed point theorem for mapping satisfying a contractive condition of rational type in partially ordered $S$-metric space. Our result generalize some existing results in the literature into settings of $S$-metric space.

\section{Introduction}

Fixed point theory is one of the most powerful and fruitful tools in nonlinear analysis. Its core subject is concerned with the conditions for the existence of one or more fixed points of a mapping $T$ from a topological space $X$ into itself; that is, we can find $x \in X$ such that $T x=x$. The Banach's contraction principle ensures under appropriate conditions the existence and uniqueness of a fixed point. It is the simplest and one of the most important results in fixed point theory. Many authors have extended, improved and generalized Banach's theorem in several ways. See $[1,2,5]$.

Some well-known fixed point in partially ordered set has been considered by some authors. For example, Ran and Reurings [15], Bhaskar and Lakshmikantham [3], Lakshmikantham and Ciric [11], Neito and Lopez [14], Harjani and Sadarangani [9] and Harjani et al [8].

Because modification, enrichment and extension of domain to a more general space is one of the active research in fixed point, some authors have tried to give generalization of metric space in several ways. For example, 2-metric space introduced by Gähler [7], $D$-metric space by Dhage [6], $G$-metric space by Mustafa and Sims [12], $D^{*}$-metric space by Sedghi et al [16] and $S$-metric space by Sedghi et al [17].

Sedghi et al [17] introduced a new generalized metric space called an $S$-metric space.

Definition 1.1. Let $X$ be a nonempty set. An $S$-metric space on $X$ is a function $S: X^{3} \rightarrow[0, \infty)$ that satisfies the following conditions. For each $x, y, z, a \in X$

(S1) $S(x, y, z) \geq 0$ for all $x, y, z \in X$

(S2) $S(x, y, z)=0$ if and only if $x=y=z$

(S3) $S(x, y, z) \leq S(x, x, a)+S(y, y, a)+S(z, z, a)$

Example 1.1 (See [17]). Let $\mathbb{R}$ be the real line. Then $S(x, y, z)=|x-z|+|y-z|$ for all $x, y, z \in \mathbb{R}$ is an $S$-metric on. This $S$-metric is called the usual $S$-metric on $\mathbb{R}$.

Example 1.2 (See [17]) Let $X=\mathbb{R}^{2}$ and $d$ an ordinary metric on $X$. Then

$S(x, y, z)=d(x, y)+d(x, z)+d(y, z)$ is an $S$-metric on $X$.

Example 1.3 (See [17]) Let $X=\mathbb{R}^{n}$ and $\|$. $\|$ a norm on $X$. Then

$S(x, y, z)=\|y+z-2 x\|+\|y-z\|$ is a $S$-metric on $X$.

Definition 1.2. (See [17]) Let $(X, S)$ be a $S$-metric space.

(1) A sequence $\left\{x_{n}\right\}, x \in X$ converges to $x$ if and only if $S\left(x_{n}, x_{n}, x\right) \rightarrow 0$ as $n \rightarrow \infty$. That is for each $\epsilon>0$ there exist $n_{0} \in \mathbb{N}$ such that for all $n \geq n_{0}, S\left(x_{n}, x_{n}, x\right)<\epsilon$ and we denote this bylim $\lim _{n \rightarrow \infty} x_{n}=x$.

(2) A sequence $x_{n}$ is called a Cauchy sequence if for each $\epsilon>0$, there exist $n_{0} \in \mathbb{N} \in$ such that $S\left(x_{n}, x_{n}, x_{m}\right)<\epsilon$ for each $n, m \geq n_{0}$

(3) The $S$-metric space $(X, S)$ is said to be complete if every Cauchy sequence converges. 
Lemma 1.1 (See [17]) Let $(X, S)$ be an $S$-metric space. Then we have

$$
S(x, x, y)=S(y, y, x)
$$

Lemma 1.2 (See [17]) Let $(X, S)$ be an $S$-metric space. Then

$$
S(x, x, z) \leq 2 S(x, x, y)+S(y, y, z)
$$

Lemma 1.3 (See [17]) Let $(X, S)$ be an $S$-metric space. If the sequence $\left\{x_{n}\right\} \in X$ converges to $x$, then $\left\{x_{n}\right\}$ is a Cauchy sequence.

Jaggi [10] proved the following theorem.

Theorem 1.1. Let $T$ be a continuous selfmap defined on a complete metric space $(X, d)$. Suppose that $T$ satisfies the following contractive condition:

$$
d(T x, T y) \leq \alpha \cdot \frac{d(x, T x) \cdot d(y, T y)}{d(x, y)}+\beta \cdot d(x, y),
$$

for all $x, y \in X, x \neq y$, and for some $\alpha, \beta \in[0,1)$ with $\alpha+\beta<1$, then $T$ has a unique fixed point in $X$.

In [8] Harjani et al gave a version of Theorem 1.1 in partially ordered metric space as follows.

Theorem 1.2 Let $(X, \leq)$ be a partially ordered set and suppose that there exist a metric $d$ in $X$ such that $(X, d)$ is a complete metric space. Let $T: X \rightarrow X$ be a continuous and nondecreasing mapping such that

$$
d(T x, T y) \leq \alpha \cdot \frac{d(x, T x) \cdot d(y, T y)}{d(x, y)}+\beta \cdot d(x, y)
$$

for $x, y \in X, x \neq y$, and for some $\alpha, \beta \in[0,1)$ with $\alpha+\beta<1$. If their exists $x_{0}<T x_{0}$ then $T$ has a fixed point in $X$.

The main aim of this paper is to generalize the result of Harjani et al [8] in the settings of $S$-metric space.

\section{Main Result}

Definition 1.1 Let $(X, \leq)$ be a partially ordered set and $T: X \rightarrow X$. We say that $T$ is a nondecreasing mapping if for $x, y \in X, x \leq y \Rightarrow T x \leq T y$.

Theorem 2.1. Let $(X, \leq)$ be a partially ordered set and $(X, S)$ is a complete $S$-metric space. Let $T: X \rightarrow X$ be a continuous and nondecreasing mapping such that

$$
S(T x, T x, T y) \leq \alpha \frac{S(x, x, T x) \cdot S(y, y, T y)}{S(x, x, y)}+\beta S(x, x, y),
$$

for $x, y \in X, x \neq y$, and for some $\alpha, \beta \in[0,1)$ with $\alpha+\beta<1$. If their exists $x_{0}<T x_{0}$ then $T$ has a fixed point in $X$.

Proof. If $T x_{0}=x_{0}$, then the proof is finished. Suppose that $x_{0}<T x_{0}$. Since $T$ is a nondecreasing mapping, we obtain by induction

$$
x_{0}<T x_{0} \leq T^{2} x_{0} \leq \ldots \leq T^{n} x_{0} \leq T^{n+1} x_{0} \leq \ldots
$$


Let $x_{n+1}=T x_{n}$. Suppose there exists $n \geq 1$ such that $x_{n+1}=x_{n}$. If $x_{n+1}=T x_{n}=x_{n}$, then $x_{n}$ is a fixed point. So we assume that $x_{n+1} \neq x_{n}$.

Putting $x=x_{n}$ and $y=x_{n-1}$ in (2.1), we get for $n \geq 1$,

$$
\begin{aligned}
S\left(x_{n+1}, x_{n+1}, x_{n}\right) & =S\left(T x_{n}, T x_{n}, T x_{n-1}\right) \\
& \leq \alpha \frac{S\left(x_{n}, x_{n}, T x_{n}\right) \cdot S\left(x_{n-1}, x_{n-1}, T x_{n-1}\right)}{S\left(x_{n}, x_{n}, x_{n-1}\right)}+\beta S\left(x_{n}, x_{n}, x_{n-1}\right) \\
& =\alpha \frac{S\left(x_{n}, x_{n}, x_{n+1}\right) \cdot S\left(x_{n-1}, x_{n-1}, x_{n}\right)}{S\left(x_{n}, x_{n}, x_{n-1}\right)}+\beta S\left(x_{n}, x_{n}, x_{n-1}\right) \\
& =\alpha S\left(x_{n}, x_{n}, x_{n+1}\right)+\beta S\left(x_{n}, x_{n}, x_{n-1}\right) .
\end{aligned}
$$

Hence we have

$$
S\left(x_{n+1}, x_{n+1}, x_{n}\right) \leq \frac{\beta}{1-\alpha} S\left(x_{n}, x_{n}, x_{n-1}\right) .
$$

By induction we have for $n \geq 0$, we get

$$
\begin{aligned}
S\left(x_{n+1}, x_{n+1}, x_{n}\right) & \leq \frac{\beta}{1-\alpha} S\left(x_{n}, x_{n}, x_{n-1}\right) \\
& \leq\left(\frac{\beta}{1-\alpha}\right)^{2} S\left(x_{n-1}, x_{n-1}, x_{n-2}\right) \\
& \cdot \\
& \cdot \\
& \leq\left(\frac{\beta}{1-\alpha}\right)^{n} S\left(x_{1}, x_{1}, x_{0}\right) .
\end{aligned}
$$

Moreover by lemma 1.2, we have for all $n \leq m$

$$
\begin{aligned}
S\left(x_{n}, x_{n}, x_{m}\right) \leq & \left(2 S\left(x_{n}, x_{n}, x_{n+1}\right)+S\left(x_{n+1}, x_{n+1}, x_{n}\right)\right) \\
\leq & \left(2 S\left(x_{n}, x_{n}, x_{n+1}\right)+2 S\left(x_{n+1}, x_{n+1}, x_{n}\right)\right) \\
& \quad+\ldots+2 S\left(x_{m-2}, x_{m-2}, x_{m-1}\right)+\left(S\left(x_{m-1}, x_{m-1}, x_{m}\right)\right. \\
\leq & \left(2 S\left(x_{n}, x_{n}, x_{n+1}\right)+\ldots+\left(2 S\left(x_{m-1}, x_{m-1}, x_{m}\right)\right)\right. \\
\leq & 2\left[\left(\frac{\beta}{1-\alpha}\right)^{n}+\left(\frac{\beta}{1-\alpha}\right)^{n+1}+\ldots+\left(\frac{\beta}{1-\alpha}\right)^{m-1}\right]\left(S\left(x_{0}, x_{0}, x_{1}\right)\right) \\
\leq & \frac{2\left(\frac{\beta}{1-\alpha}\right)^{n}}{1-\left(\frac{\beta}{1-\alpha}\right)}\left(S\left(x_{1}, x_{1}, x_{0}\right)\right) .
\end{aligned}
$$

Since $\left(\frac{\beta}{1-\alpha}\right)<1$, taking limit as $n, m \rightarrow \infty$, we get

$$
\lim _{n, m \rightarrow \infty} S\left(x_{n}, x_{n}, x_{m}\right)=0
$$

Therefore $\left\{x_{n}\right\}$ is a Cauchy sequences in $X$.

Since $X$ is complete, there exist $x^{*} \in X$ such that as $n \rightarrow \infty, x_{n} \rightarrow x^{*}$.

Also the continuity of $T$ implies

$$
T x^{*}=T\left(\lim _{n \rightarrow \infty} x_{n}\right)=\lim _{n \rightarrow \infty} T x_{n}=\lim _{n \rightarrow \infty} x_{n+1}=x^{*},
$$

which proves that $x^{*}$ is a fixed point. 
In the next theorem, we omit the continuity of $T$ and assume the following hypothesis in $X$. This hypothesis has been stated in [8].

C1: If $\left\{x_{n}\right\}$ is a nondecreasing sequence such that $x_{n} \rightarrow x$, then $x=\sup \left\{x_{n}\right\}$.

Theorem 2.2. Let $(X, \leq)$ be a partially ordered set and $(X, S)$ is a complete $S$-metric space. Assume that $X$ satisfies $(C 1)$. Let $T: X \rightarrow X$ be a continuous and nondecreasing mapping such that

$$
S(T x, T x, T y) \leq \alpha \frac{S(x, x, T x) \cdot S(y, y, T y)}{S(x, x, y)}+\beta S(x, x, y)
$$

for $x, y \in X, x \neq y, x \geq y$ and for some $\alpha, \beta \in[0,1)$ with $\alpha+\beta<1$. If their exists $x_{0}<T x_{0}$ then $T$ has a fixed point in $X$.

Proof. Following the proof of Theorem 2.1, we only need to verify $T x^{*}=x^{*}$.

Since $\left\{x_{n}\right\}$ is a nondecreasing sequence in $X$ and $x_{n} \rightarrow x^{*}$, then by (C1) with $x_{n} \leq x^{*}$ and for all $n \in \mathbb{N}, x^{*}=\sup \left\{x_{n}\right\}$.

Since $T$ is a nondecreasing mapping, then $T x_{n} \leq T x^{*}$ or $x_{n+1} \leq T x^{*}$ for all $n \in \mathbb{N}$.

Moreover, as $x_{0}<x_{1} \leq T x^{*}$ and $x^{*}=\sup \left\{x_{n}\right\}$, we get $x^{*} \leq T x^{*}$.

Suppose that $x^{*}<T x^{*}$. Using similar argument as in the proof of Theorem 2.1, for $x_{0} \leq T x_{0}$, we obtain that $\left\{T^{n} x^{*}\right\}$ is a nondecreasing sequence and $\lim _{n \rightarrow \infty} T^{n} x^{*}=z$ for some $z \in X$.

Again using (C1), we get that $v=\sup \left\{T^{n} x^{*}\right\}$. Moreover from $x_{0} \leq x^{*}$, we get $x_{n}=T^{n} x_{0} \leq T^{n} x^{*}$ for $n \geq 1$ and $x_{n}<T^{n} x^{*}$ because $x_{n} \leq x^{*} \leq T x^{*} \leq T^{n} x^{*}$ for $n \geq 1$.

As $x_{n}$ and $T^{n} u$ are comparable and distinct for $n \geq 1$, applying (2.1) we get

$$
\begin{aligned}
S\left(x_{n+1}, x_{n+1}, T^{n+1} x^{*}\right) & =S\left(T x_{n}, T x_{n}, T\left(T^{n} x^{*}\right)\right) \\
& \leq \alpha \frac{S\left(x_{n}, x_{n}, T x_{n}\right) \cdot S\left(T^{n} x^{*}, T^{n} x^{*}, T^{n+1} x^{*}\right)}{S\left(x_{n}, x_{n}, T^{n} x^{*}\right)}+\beta S\left(x_{n}, x_{n}, T^{n} x^{*}\right) \\
& \leq \alpha \frac{S\left(x_{n}, x_{n}, x_{n+1}\right) \cdot S\left(T^{n} x^{*}, T^{n} x^{*}, T^{n+1} x^{*}\right)}{S\left(x_{n}, x_{n}, T^{n} x^{*}\right)}+\beta S\left(x_{n}, x_{n}, T^{n} x^{*}\right)
\end{aligned}
$$

Letting $n \rightarrow \infty$ in the above inequality, we obtain

$$
S\left(x^{*}, x^{*}, z\right) \leq \beta S\left(x^{*}, x^{*}, z\right) .
$$

As $\beta<1, S\left(x^{*}, x^{*}, z\right)=0$, thus $x^{*}=z$.

Particularly $x^{*}=z=\sup \left\{x_{n}\right\}$ and therefore $T x^{*} \leq x^{*}$ and thus we have a contradiction.

Hence $T x^{*}=x^{*}$.

For the uniqueness of the fixed point, we consider the following condition stated in [8].

(C2): For $x, y \in X$, there exists $u \in X$ which is comparable to $x$ and $y$.

Theorem 2.3. Adding (C2) to the hypotheses of Theorem 2.1 (or Theorem 2.2) one obtains the uniqueness of the fixed point.

Proof. Suppose that $x^{*}, y^{*} \in X$ are fixed points. We consider two cases. 
Case 1. If $x^{*}$ and $y^{*}$ are comparable and $x^{*} \neq y^{*}$, then using the contractive condition we have

$$
\begin{aligned}
S\left(x^{*}, x^{*}, y^{*}\right) & =S\left(T x^{*}, T x^{*}, T y^{*}\right) \\
& \leq \alpha \frac{S\left(x^{*}, x^{*}, T x^{*}\right) \cdot S\left(y^{*}, y^{*}, T y^{*}\right)}{S\left(x^{*}, x^{*}, y^{*}\right)}+\beta S\left(x^{*}, x^{*}, y^{*}\right) \\
& \leq \alpha \frac{S\left(x^{*}, x^{*}, x^{*}\right) \cdot S\left(y^{*}, y^{*}, y^{*}\right)}{S\left(x^{*}, x^{*}, y^{*}\right)}+\beta S\left(x^{*}, x^{*}, y^{*}\right) \\
& =\beta S\left(x^{*}, x^{*}, y^{*}\right) .
\end{aligned}
$$

Hence $S\left(x^{*}, x^{*}, y^{*}\right) \leq \beta S\left(x^{*}, x^{*}, y^{*}\right)$.

Since $\beta<1$ in (2.5), it implies a contradiction. Therefore $x^{*}=y^{*}$.

Case 2. If $x^{*}$ is not comparable to $y^{*}$, then by (C2) there exists $x \in X$ comparable to $x^{*}$ and $y^{*}$. Monotonicity implies that $T^{n} x$ is comparable to $T^{n} x^{*}=x^{*}$ and $T^{n} y^{*}=y^{*}$ for $n \geq 0$.

If there exist $n_{0} \geq 1$ such that $T^{n_{0}} x=x^{*}$, then as $x^{*}$ is a fixed point, the sequence $\left\{T^{n} x: n \geq n_{0}\right\}$ is constant and consequently $\lim _{n \rightarrow \infty} T^{n} x=x^{*}$.

On the other hand, if $T^{n} x \neq x^{*}$ for $n \geq 1$, using contractive condition, we obtain for $n \geq 2$,

$$
\begin{aligned}
& S\left(T^{n} x, T^{n} x, x^{*}\right)=S\left(T^{n} x, T^{n} x, T^{n} x^{*}\right) \\
& \leq \alpha \frac{S\left(T^{n-1} x, T^{n-1} x, T^{n} x^{*}\right) \cdot S\left(T^{n-1} x^{*}, T^{n-1} x^{*}, T^{n} x^{*}\right)}{S\left(T^{n-1} x, T^{n-1} x, T^{n-1} x^{*}\right)}+\beta S\left(T^{n-1} x, T^{n-1} x, T^{n-1} x^{*}\right) \\
& \quad \leq \alpha \frac{S\left(T^{n-1} x, T^{n-1} x, T^{n} x^{*}\right) \cdot S\left(x^{*}, x^{*}, x^{*}\right)}{S\left(T^{n-1} x, T^{n-1} x, x^{*}\right)}+\beta S\left(T^{n-1} x, T^{n-1} x, x^{*}\right) \\
& \quad=\beta S\left(T^{n-1} x, T^{n-1} x, x^{*}\right) .
\end{aligned}
$$

Hence $S\left(T^{n} x, T^{n} x, x^{*}\right) \leq \beta S\left(T^{n-1} x, T^{n-1} x, x^{*}\right)$.

Using induction, for $n \geq 2$, we have

$$
S\left(T^{n} x, T^{n} x, x^{*}\right) \leq \beta^{n} S\left(x, x, x^{*}\right) .
$$

Now, as $\beta<1$, we have $\lim _{n \rightarrow \infty} T^{n} x=x^{*}$.

In a similar manner one can show that $\lim _{n \rightarrow \infty} T^{n} x=y^{*}$.

This shows that $x^{*}=y^{*}$ and hence the fixed point is unique.

\section{REFERENCES}

[1] R. P. Agarwal, M. A. El-Gebeily, and D. O'Regan, Generalized contractions in partially ordered metric spaces, Applicable Analysis, 87(1) (2008), 109-116.

[2] D. Ariza-Ruiz, A. Jimnez-Melado, A continuation method for weakly contractive mappings under the interior condtions. Fixed Point Theory Appl. 2009 (2009) Article ID 809315.

[3] T.G. Bhaskar and V. Lakshmikantham, Fixed point theorems in partially ordered metrispaces and applications, Nonlinear Anal., 65(2006), 1379-1393.

[4] V. Berinde and M. Borcut, Tripled fixed point theorems for contractive type mappings in partially ordered metric spaces, Nonlinear Anal., 74(15) (2011), 4889-4897.

[5] S. K., Chatterjea, Fixed point theorems, C. R. Acad.Bulgare Sci., 25 (1972) 727 - 730. 
[6] B. C. Dhage, A study of some fixed point theorems, Ph.D. Thesis, Marathwada Univ. Aurangabad, India 1984.

[7] S. Gähler, 2-metriche raume und ihre toplogische strukture. Maths.Nachr. , 26 (1963) 115-148.

[8] J. Harjani, B. Lopéz and K. Sadarangani, A fixed point theorems for mappings satisfying a contractive condition of rational type on a partially ordered metric spaces, Abstract and Applied Analysis, (2010), Article ID 190701.

[9] J. Harjani and K. Sadarangani, Fixed point theorems for weakly contractive mappings in partially ordered sets, Nonlinear Analysis: Theory, Methods \& Applications, 71(7) (2009), 3403-3410.

[10]D. S. Jaggi, Some unique fixed point theorems, Indian Journal of Pure and Applied Mathematics, vol. 8, no. 2, pp. 223-230, 1977.

[11] V. Lakshmikantham and L. Ciric, Coupled fixed point theorems for nonlinear contractions in partially ordered metric spaces, Nonlinear Anal., 70(2009), 4341-4349.

[12]Z. Mustafa and B. Sims, A new approach to generalize metric space, J. Roy.nonlinear convex anal. 7(2006) $289-297$.

[13]Z. Mustafa, H. Obiedat and F. Awawdeh, Some common fixed point theorems for mapping on complete G-metric space, Fixed Point Theory Appl.2008 (2008) Article ID 189870.

[14] J.J. Nieto and R.R. Lopez, Existence and uniqueness of fixed point in partially ordered sets and applications to ordinary differential equations, Acta Mats. Sinica (Engl. Ser.), 23(12) (2007), 2205-2212.

[15]A.C.M. Ran and M.C.B. Reurings, A fixed point theorem in partially ordered sets and some applications to metric equations, Proc. Amer. Math. Soc., 132(2004), 1435-1443.

[16] S. Sedghi, N. Shobe and H. Zhou, A common fixed point theorem in $D^{*}$-metric space, Fixed Point Theory Appl.2007 (2007) Article ID 027906.

[17] S. Sedghi, N. Shobe and A. Aliouche, A generalization of fixed point theorem in $S$-metric space, Mat.Vesinik. 64(3), (2012) 258 -266. 